\title{
Mössbauer study of the hyperfine interactions and spin dynamics in $\alpha$-iron(II) phthalocyanine
}

\author{
G. Filoti \\ National Institute for Materials Physics, P.O. Box MG-07, 77125 Bucharest-Magurele, Romania, \\ and Instituto de Ciencia de Materiales de Aragón, CSIC-Universidad de Zaragoza, 50009 Spain
}

M. D. Kuz'min* and J. Bartolomé

Instituto de Ciencia de Materiales de Aragón, CSIC-Universidad de Zaragoza, 50009 Spain

(Received 22 December 2005; revised manuscript received 7 April 2006; published 20 October 2006)

\begin{abstract}
The ${ }^{57} \mathrm{Fe}$ Mössbauer spectroscopy on $\alpha$-iron(II) phthalocyanine (FePc) as a function of temperature (1.3 $<\mathrm{T}<295 \mathrm{~K})$ and applied field $(0<\mathrm{B}<10 \mathrm{~T})$ has been used to study the peculiar magnetic properties of this ferromagnetic quasilinear chain type compound. One sextet with an internal hyperfine field $B_{\text {int }}=66.2 \mathrm{~T}$ was observed at $1.3 \mathrm{~K}$, a very large value for a bivalent iron with $S=1$ pointing to the existence of large positive orbital and dipolar contributions in the investigated FePc. Under an applied field, the experimental spectra exhibited two nonequivalent Fe positions, due to spin canting, with the values for the hyperfine fields of the split sextets increasing with increasing field, an indication that unlike most cases, $B_{\text {int }}$ in $\alpha$-FePc is positive, i.e., parallel to the magnetic moment of iron. Therefore, the origin of the large hyperfine field is the orbital moment rather than the Fermi's contact interaction. This fact is ascribed to the orbital degeneracy of the ground state of $\mathrm{Fe}(\mathrm{II})$ in the present configuration, where an unpaired hole occupies the orbital doublet $\left(d_{x z}, d_{y z}\right)$. This feature supports and explains the magnetization and susceptibility data as well as the anomalously high hyperfine field observed at ${ }^{57} \mathrm{Fe}$ nucleus. The relaxational behavior in the ac susceptibility and Mössbauer spectra found in the region 5-20 K was ascribed to solitonlike motion of domain walls within the magnetic chains, with a singlekink activation energy of $72 \mathrm{~K}$.
\end{abstract}

DOI: $10.1103 /$ PhysRevB.74.134420

PACS number(s): 75.50.Xx, 76.80.+y, 75.50.Dd

\section{INTRODUCTION}

The metal phthalocyanines (MPc) form a family of compounds with a wide range of commercial applications such as catalysts or dyes, and more recently in thin film technology because of their electro-optical properties. ${ }^{1}$ Among them iro$\mathrm{n}(\mathrm{II})$ phthalocyanine $(\mathrm{FePc})$ attracted attention as a test molecule close to the iron (haem) proteins because of their structural similarity, ${ }^{2}$ the FePc acting as a model system. The initial interest on this work was stimulated by the potential use of MPc as a gas sensor, ${ }^{3}$ where an acceptor-donor mechanism could appear under irradiation, as in the case of doped polymer media for real time holography. ${ }^{4,5}$ From a more basic point of view, the $\mathrm{MnPc}$ was one of the first molecular magnets reported. Indeed, magnetic properties were determined on a single crystal specimen. ${ }^{6}$ Because of the interest in molecular magnetism, in a previous paper the $\alpha-\mathrm{FePc}$ magnetic properties were studied in detail, finding ferromagnetic behavior below $10 \mathrm{~K} .^{7}$ Mössbauer spectroscopy has revealed a very large and unusual positive hyperfine field at the ${ }^{57} \mathrm{Fe}$ nucleus, a record for $\mathrm{Fe}$ compounds with intermediate spin $S=1$. This paper reports on this peculiar experimental behavior and provides strong arguments that this feature is derived from a highly orbital degenerate ground state.

The metal-free phthalocyanine presents two available electrons (two hydrogen atoms) connected via direct bond with only two, out of four available, pyrrolic $\mathrm{N}$ atoms in a well-described planar configuration of tetragonal local molecular symmetry. There are many metal-phthalocyanine compounds of the $3 d$ transition period elements, such as $\mathrm{Cu}$, $\mathrm{Co}, \mathrm{Mn}, \mathrm{Mg}$, or Fe, which form solid structures of the so- called herringbone type. They consist of linear chains of stacked planar molecules with the fourfold axis of the individual molecule forming an angle with the crystal $b$ axis. The adjacent chains are inclined in the opposite sense. There are several types of phases, depending on the angle and the shift along the crystallographic $b$ axis of one linear chain with respect to the other. ${ }^{8}$ In particular, FePc crystallizes in the $\alpha$ (Ref. 9) and $\beta$ (Ref. 10) forms. Their different stacking angles vary the coordination of the $\mathrm{Fe}$ atom and $\mathrm{Fe}-\mathrm{Fe}$ distance along the $b$ axis, and consequently, give rise to different magnetic properties.

The magnetic susceptibility from room temperature down to about $100 \mathrm{~K}$ of both the $\alpha$ - and $\beta$-FePc phases obey an identical paramagnetic Curie-Weiss law, which can be described within the $S=1$ effective spin model, albeit with a high value of $g=2.4$ that is indicative of orbital moment contribution. At lower temperatures the curves diverge: while the $\beta$-FePc behaves as a singlet-ground-state paramagnet down to the lowest temperatures, ${ }^{11-14}$ the $\alpha$-FePc susceptibility increases rapidly with decreasing temperature in close resemblance to the Ising linear chain behavior down to about $10 \mathrm{~K} .{ }^{7}$ Below $10 \mathrm{~K}$ the ac susceptibility and magnetization data proved the existence of ferromagnetism, although the long range ordering peak could not be evidenced from heat capacity experiments, not very surprisingly in view of the very fine powder form of the sample and the chainlike magnetic behavior. However, an interesting feature remains unexplained. In the remanence versus temperature measurements, ac susceptibility and field cooled and zero field cooled (FC-ZFC) magnetization measurements, there is a second crossover temperature at about $5 \mathrm{~K}$ to a very sluggish irreversible magnetic phase. 
To clarify this puzzling behavior, we have performed a careful Mössbauer spectroscopy study both as a function of temperature and an external applied field to check whether we could observe the crossover from the paramagnetic to the ordered regime through this relaxation region.

Thirty years ago, a remarkable number of Mössbauer, ${ }^{15-18}$ magnetic, ${ }^{13,19}$ and structural ${ }^{20,21}$ investigations were performed on FePc, but it has to be noticed that the quoted data have shown a quite large dispersion range of the reported values and properties. Most of them, when not certainly identified, were performed on $\beta$-FePc samples. The isomer shift $(\delta)$ showed differences up to $0.18 \mathrm{~mm} / \mathrm{sec}$ and the quadrupole splitting $\left(\Delta E_{Q}\right)$ comparable variation of $0.19 \mathrm{~mm} / \mathrm{sec}$ at $80 \mathrm{~K}$. The magnetic data were discussed in terms of the appropriate electronic level populations as well as of potential orbital contributions to the magnetic properties. One reason for the existence of such controversial data could originate from the fact that, in most cases, each report dealt with its own freshly prepared samples, often on samples with an ill-defined structure, morphology, defects, interactions, or magnetic properties.

Previous Mössbauer spectroscopy studies consisted of a comparative analysis of the $\alpha$-FePc and $\beta$-FePc paramagnetic doublet at $80 \mathrm{~K},{ }^{22}$ or with pressure, ${ }^{23}$ at varying temperatures ${ }^{24}$ and in thin films. ${ }^{25}$ The main conclusion of those studies ${ }^{22-24}$ was that the $\delta$ and $\Delta E_{Q}$ of $\alpha$-FePc are smaller than those of $\beta$-FePc by a maximum of 0.04 and, respectively, $0.16 \mathrm{~mm} / \mathrm{sec}$, at room temperature. It reflects the different coordination of the Fe with the adjacent molecules. In the $\beta$ form the $\mathrm{N}$ atoms from the neighboring molecules are present in apical octahedral positions, while in the $\alpha$ form they are not in axial positions and do not form a $\mathrm{N}$ octahedron around the central Fe. ${ }^{22}$ The first Mössbauer study of $\beta$-FePc identified the $\mathrm{Fe}$ in this molecule as of intermediate spin $S=1{ }^{26}$ Further on, the low-temperature Mössbauer spectroscopy studies were done on the $\beta$-FePc. They proved that the $\mathrm{Fe}$ atom located in the square-planar coordination of FePc behaved as an $S=1$ effective spin system, and, in agreement with magnetic measurements, no trace of ordering (no sextet) could be detected in the Mössbauer spectra. ${ }^{27}$

More recently, on the other hand, there is an $S=1$ compound $\mathrm{Fe}(\mathrm{II})$ octaethyltetraazaporphyrin (FeOETAP), which is also a square-planar molecule that crystallizes in herringbone structure, that showed a Mössbauer sextet at lower temperature, ${ }^{28,29}$ from which an unusual large value of the hyperfine field $B_{\text {int }}=62.4 \mathrm{~T}$ was derived. In a previous paper on $\alpha$-FePc (Ref. 7) preliminary low-temperature Mössbauer data were mentioned that showed a clear sextet pattern below $10 \mathrm{~K}$, which resulted in a still higher $B_{\text {int }}=66.2 \mathrm{~T}$ at $1.3 \mathrm{~K}$. This is a record value for an effective $S=1$ system. In this paper, a detailed low-temperature Mössbauer spectroscopic study of $\alpha$-FePc involving both temperature and "in-field" data is reported.

\section{EXPERIMENTAL DETAILS}

The experiments were performed on powder samples of $\alpha-\mathrm{Fe}(\mathrm{Pc})$ compound commercially available from Aldrich
Co. (Catalogue no. $=37,954.9)$. It is worth noting that scanning electron microscopy (SEM) revealed needle-shape morphology with particles stacked as a bunch of rods, having a ratio of 10 between length and thickness. When deposited on a flat surface, it was directly seen by optical microscopy that the needles have the habit of lying nearly parallel to that surface, with their long axes randomly oriented in the plane. From powder x-ray diffraction data, ${ }^{7}$ the structure could be classified as $\alpha I$ form, ${ }^{8}$ and from the cell dimensions it was concluded that the molecules are stacked parallel to each other along the short $b$ axis at intervals of $3.78 \AA$, and the molecular planes are inclined at an angle of $26.5^{\circ}$ with respect to the $a c$ plane, ${ }^{7}$ in good agreement with the previous x-ray data. ${ }^{9}$

The temperature dependent Mössbauer measurements have been performed using a helium bath cryostat between $4.2 \mathrm{~K}$ and room temperature. The linear symmetrical waveform mode of the velocity was utilized. The ${ }^{57} \mathrm{Co}$ source in rhodium matrix had an average activity of $1 \mathrm{GBq}$. An $\alpha$-Fe foil was used for calibration, and the $\delta$ is permanently referred to it. The absorber powder was stacked in a $\mathrm{Cu}$ holder, strongly pressed between two mica foils. The thickness of the absorber was of about $13.5 \mathrm{mg} \mathrm{Fe} / \mathrm{cm}^{2}$, a value higher than the traditional one of $10 \mathrm{mg} \mathrm{Fe} / \mathrm{cm}^{2}$, inducing a little but noticeable larger linewidth. The reason for an increased thickness was related to the extended distance between source and detector in the experimental setup, in order to obtain an increased absorption effect. The measurements in applied fields up to 10 Tesla (T) (in the direction of $\gamma$ rays, i.e., perpendicular to the sample holder plane) as well as the zero-field measurements at the lowest temperature $1.3 \mathrm{~K}$ were performed at the Department of Physics, Liverpool University, with an Oxford in-field bath cryostat, using a sawtooth wave form for the relative velocity between source and absorber. The data at Liverpool University (the $1.3 \mathrm{~K}$ one and those in applied fields) were acquired on a double number of channels (512 in the folded spectra) than the ones measured in the He-bath cryostat (all the other spectra versus temperature) as could be observed from data presented in the following pictures.

The fitting procedure involved a nonlinear regression method using Lorentz line shapes and one common linewidth per pattern (doublet or sextet), as presented in Tables I and II.

Magnetic measurements were performed in a superconducting quantum interference device (SQUID) magnetometer, with an ac susceptibility option. The complex ac susceptibility on a randomly oriented powder at frequencies ranging from $1 \mathrm{~Hz}$ to $1 \mathrm{KHz}$, and bias field $(H)$ up to $0.1 \mathrm{~T}$ was also measured.

\section{SPECTRA ANALYSIS}

\section{A. Temperature dependent measurements}

Most of the Mössbauer spectra at zero field recorded at various temperatures are shown in Fig. 1 (but one at $4.2 \mathrm{~K}$ presented in Fig. 3). At the lowest temperature of $1.3 \mathrm{~K}$ and at $4.2 \mathrm{~K}$, the spectra exhibit one sextet with linewidth $(\Gamma)$ of $0.54 \mathrm{~mm} / \mathrm{sec}$ and, respectively, $0.56 \mathrm{~mm} / \mathrm{sec}$ (Table I), 
TABLE I. The Mössbauer results of $\alpha$-FePc with increasing temperature.

\begin{tabular}{cccccc}
\hline \hline$T$ & $B_{\text {int }}$ & $\begin{array}{c}\varepsilon \\
(\mathrm{mm} / \mathrm{sec})\end{array}$ & $\begin{array}{c}\delta \\
(\mathrm{mm} / \mathrm{sec})\end{array}$ & $\begin{array}{c}\Gamma \\
(\mathrm{mm} / \mathrm{sec})\end{array}$ & $\begin{array}{c}\varphi \\
\left(B / V_{z z}\right)\end{array}$ \\
\hline $1.3 \pm 0.2$ & $(\mathrm{~T})$ & $-0.225 \pm 0.02$ & $0.46 \pm 0.02$ & $0.54 \pm 0.02$ & $51.2^{\circ}$ \\
$4.2 \pm 0.2$ & $66.20 \pm 0.04$ & $-0.225 \pm 0.02$ & $0.46 \pm 0.02$ & $0.56 \pm 0.02$ & $51.2^{\circ}$ \\
$7.5 \pm 0.2$ & $65.91 \pm 0.09$ & $-0.218 \pm 0.03$ & $0.46 \pm 0.03$ & $0.83 \pm 0.06$ & \\
$10 \pm 0.2$ & $65.2 \pm 0.31$ & $-0.165 \pm 0.06$ & $0.45 \pm 0.05$ & $2.86 \pm 0.18$ & \\
$10 \pm 0.2$ & & $\Delta E_{Q}(\mathrm{~mm} / \mathrm{sec})$ & & \\
$25 \pm 0.2$ & 0 & $2.60 \pm 0.04$ & $0.44 \pm 0.04$ & $0.69 \pm 0.06$ & \\
$140 \pm 0.4$ & 0 & $2.58 \pm 0.02$ & $0.43 \pm 0.02$ & $0.65 \pm 0.03$ & \\
$295 \pm 0.4$ & 0 & $2.54 \pm 0.02$ & $0.41 \pm 0.02$ & $0.38 \pm 0.02$ & \\
\hline \hline
\end{tabular}

Note: The parameters of collapsed magnetic patterns were not introduced

rather normal for an iron coordination compound in the region of the magnetic order. A relaxation process develops continuously, showing at $7.5 \mathrm{~K}$ a linewidth of $0.83 \mathrm{~mm} / \mathrm{sec}$, which further increases (Fig. 2) with temperature.

The sextet collapses into a purely relaxed, very broad, spectrum (Fig. 1). Already at $10 \mathrm{~K}$ part of Fe sites are showing various magnetic interactions and have collapsed in a very broad typical central doublet, while some of Fe atoms commenced to be in a paramagnetic state. These provide a separate doublet with parameters close to the hightemperature data of $\alpha$-FePc, and are affected by larger errors induced by the presence of the central doublet reflecting the collapsed interactions. In Table I the resulting data are presented. The same features are also observed at $15 \mathrm{~K}$, evolving to a typical paramagnetic doublet, which is clearly resolved at temperatures above $25 \mathrm{~K}$. Besides the main phase a minor impurity phase $(\sim 10.5 \%)$, appears in all the spectra as an extra doublet (see its presence in the spectra plot bars; Fig. 1) that for the sake of clarity is not mentioned in Tables I and II but contribute to the spectra shape both in the magnetically ordered and in the paramagnetic region. Its parameters, the quadrupole splitting $\Delta E_{Q}=1.36 \mathrm{~mm} / \mathrm{sec}$ (almost constant over the whole temperature range) and its isomer shift $\delta=0.08 \mathrm{~mm} / \mathrm{sec}$ at $4.2 \mathrm{~K}$, are not similar to any of those reported in the literature, either for the $\alpha$ or $\beta$ polymorph phases of $\mathrm{Fe}$ phthalocyanine ${ }^{15}$ or $\mathrm{Fe}(\mathrm{III})$ phthalocyanine-chloride. ${ }^{20}$ The phase remained magnetically disordered even in fields up to $10 \mathrm{~T}$.

It is remarkable that the fitted hyperfine field parameter for the main $\alpha$-FePc phase, $B_{\text {int }}(66.2 \mathrm{~T}$ at $1.3 \mathrm{~K}$ and $66.1 \mathrm{~T}$ at $4.2 \mathrm{~K}$ ), listed in Table I, represent the highest value of the internal field at an ${ }^{57} \mathrm{Fe}$ nucleus in any known magnetically ordered iron compound with $S=1$ reported in the literature up to now. Close to this value is $B_{\text {int }}=62.4 \mathrm{~T}$, for the recently reported related compound, iron (II) octa-ethyltetra-porphyrin-FeOETAP. ${ }^{28,29}$ According to our knowledge, the largest $B_{\text {int }}$ reported for the highly ionic Fe(III) spin-only compound $\mathrm{FeF}_{3}$ with three-dimensional (3D) ordering was $62.2 \mathrm{~T}$, as quoted in a review article. ${ }^{30}$

In Fig. 2 the linewidth evolution with temperature is displayed. Between $1.3 \mathrm{~K}$ and $5 \mathrm{~K}$ the spins are fully blocked. Above this temperature region there is a very sharply temperature dependent relaxation process, starting slowly with a linewidth of $0.83 \mathrm{~mm} / \mathrm{sec}$ (see Table I) at $7.5 \mathrm{~K}$ as forecast from the interpretation of the magnetic data, ${ }^{7}$ the transition from a magnetically ordered to a disordered state taking place within the $10-12 \mathrm{~K}$ interval. The blocking crossover temperature derived from the observed maximum is around $11.8 \pm 0.5 \mathrm{~K}$. Indeed, the previously reported magnetic data on the same compound also showed a slowing down of the magnetic relaxation process up to blocking at approximately the same temperature. ${ }^{7}$ The linewidth reaches a constant minimum value of $0.38 \mathrm{~mm} / \mathrm{sec}$ at higher temperatures (see also data at 50, 140, and $295 \mathrm{~K}$ in Table I), quite similar to the values found for in a number of $\mathrm{Fe}$ compounds with square planar coordination.

Usually, the fitted parameters provide the angle $\varphi$ between the $B_{\text {int }}$ and the main electrical field gradient (EFG) principal axis $\left(V_{z z}\right)$, which can be obtained from the quadrupole splitting values observed in the magnetically ordered, $\varepsilon$, and disordered (paramagnetic) region $\Delta E_{Q}$, according to the formula

TABLE II. Mössbauer fit parameters of $\alpha$-FePc, measured at $4.2 \mathrm{~K}$ in an applied field, $B_{a p p l}$.

\begin{tabular}{ccccccc}
\hline \hline i-Sextet & $\begin{array}{c}B_{\text {appl }} \\
(\mathrm{T})\end{array}$ & $\begin{array}{c}B_{\text {eff.i }} \\
(\mathrm{T})\end{array}$ & $\begin{array}{c}\varepsilon_{i} \\
(\mathrm{~mm} / \mathrm{sec})\end{array}$ & $\begin{array}{c}\delta_{i} \\
(\mathrm{~mm} / \mathrm{sec})\end{array}$ & $\begin{array}{c}\Gamma_{i} \\
(\mathrm{~mm} / \mathrm{sec})\end{array}$ & $\begin{array}{c}\varphi_{\iota} \\
\left(B / V_{z z}\right)\end{array}$ \\
\hline 1 & 5 & $72.6 \pm 0.2$ & $0.14 \pm 0.03$ & $0.43 \pm 0.04$ & $0.54 \pm 0.08$ & $56.9^{\circ}$ \\
2 & 5 & $69.3 \pm 0.2$ & $0.23 \pm 0.02$ & $0.34 \pm 0.04$ & $0.63 \pm 0.07$ & $58.6^{\circ}$ \\
1 & 10 & $76.8 \pm 0.2$ & $0.04 \pm 0.02$ & $0.43 \pm 0.04$ & $0.56 \pm 0.08$ & $55.4^{\circ}$ \\
2 & 10 & $72.6 \pm 0.2$ & $0.20 \pm 0.03$ & $0.47 \pm 0.04$ & $0.66 \pm 0.07$ & $58.1^{\circ}$ \\
\hline \hline
\end{tabular}




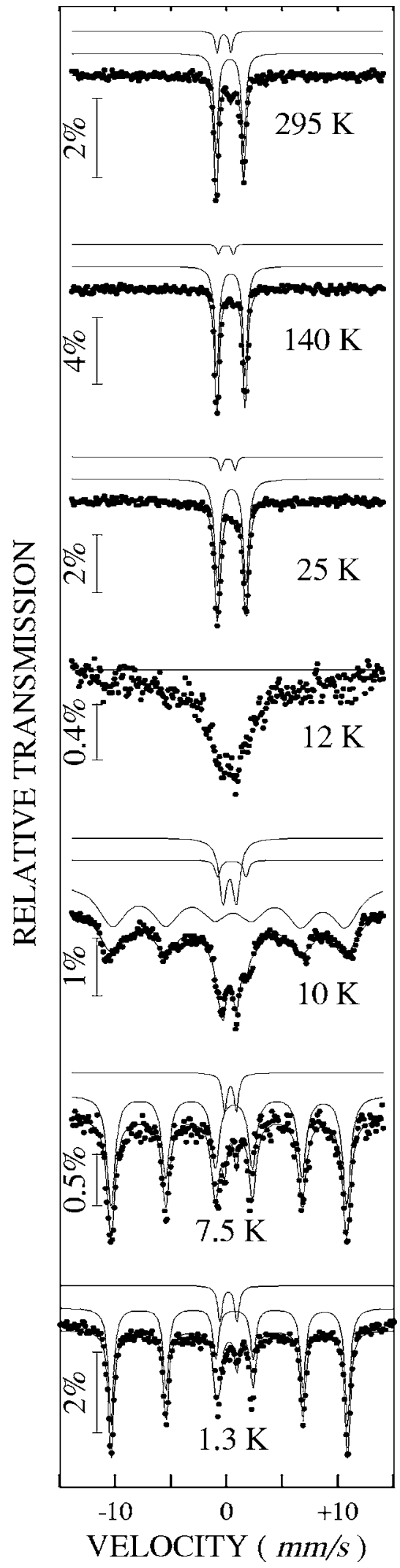

FIG. 1. The Mössbauer spectra of $\alpha-\mathrm{FePc}$ powder taken at various temperatures. Strong relaxation behavior is observed in the range 5 to $20 \mathrm{~K}$. A complete disorder state (line collapse) is fully achieved at $12 \mathrm{~K}$.

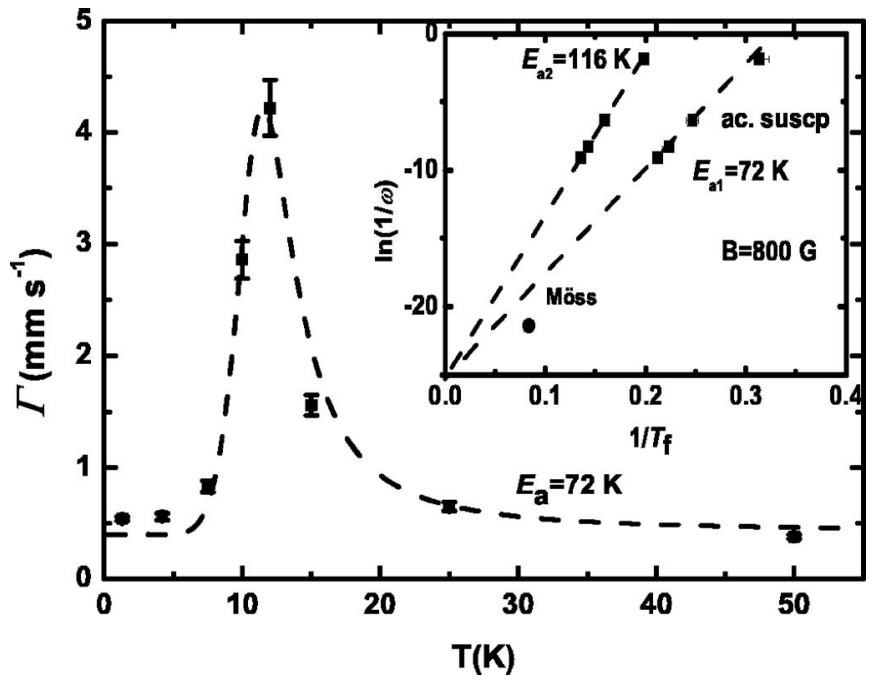

FIG. 2. Experimental linewidths $(\Gamma)$ as a function of temperature. Dashed line is a fit to the soliton single-kink contribution to the linewidth. Note the presence of an extra contribution below $5 \mathrm{~K}$, ascribed to spin-wave driven flipping modes. Inset: Inverse of the relaxation rate, on a natural logarithmic scale, vs the inverse of temperature. ( $\boldsymbol{\square})$ Relaxation rates found from the maximum of the $\chi^{\prime \prime}(T, \omega)$ peaks; $(\bigcirc)$ Relaxation rate derived from the maximum of the Mössbauer $\Gamma$ dependence on temperature.

$$
\varepsilon=1 / 2 \Delta E_{Q}\left(3 \cos ^{2} \varphi-1\right),
$$

using $\varepsilon=-0.225 \mathrm{~mm} / \mathrm{sec}$ and $\Delta E_{Q}=2.52 \mathrm{~mm} / \mathrm{sec}$ an angle $\varphi=51.2^{\circ}$ (Table I) was obtained.

The values of $\Delta E_{Q}$ and $\delta$ (Table I) above $25 \mathrm{~K}$, i.e., in the paramagnetic region, are marginally lower than the values reported earlier for other batches, ${ }^{3,15-18,22-27}$ however, they identify, especially the $\delta$ values, the divalent $\mathrm{Fe}(\mathrm{II})$ in an intermediate spin state $S=1$, in good agreement with magnetic data reported on the same compound. ${ }^{7}$ The nearly temperature independent values of $\Delta E_{Q}$ (in the limit of error) indicates a very stable electronic configuration of the molecule with no temperature dependent contribution from their own electronic shells of the bivalent iron. The isomer shift presents only a small second-order Doppler shift versus temperature also mentioned, for example, by Bell et al. ${ }^{23}$ for their sample.

\section{B. Measurements in an applied field}

The field was applied perpendicularly to the sample plane, in the same direction as the incident gamma ray. The Mössbauer data measured using applied fields of 5 and $10 \mathrm{~T}$ at 4.2 K, and without applied field, as reference, are presented in Fig. 3.

For the sake of clarity concerning the fields at the ${ }^{57} \mathrm{Fe}$ nucleus, we used the term $B_{\text {int }}$ for the spectra measured at various temperatures while the term $B_{\text {eff }}$ is the hyperfine field observed in an applied field, both referring to the effect of magnetic interactions at the nucleus. Their relationship is given by the vector sum formula

$$
\mathbf{B}_{e f f}=\mathbf{B}_{i n t}+\mathbf{B}_{a p p l}
$$




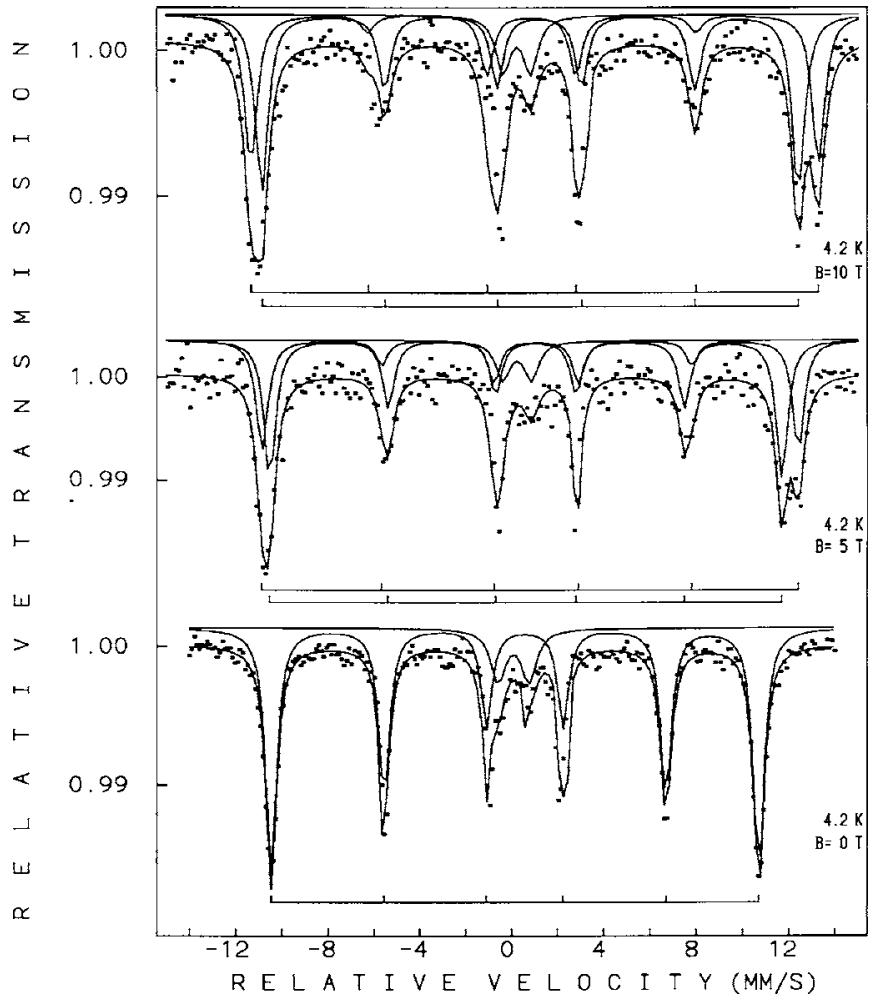

FIG. 3. Mössbauer spectra of $\alpha$-FePc taken at $4.2 \mathrm{~K}$ without field and in applied field. Full lines represent fits described in the text.

The best fits for the in-field spectra were achieved by a free iteration method with two sextets and a doublet (again representing the already mentioned impurity phase); i.e., the parameters were not constrained a priori. The most striking aspect induced by the external field is the splitting of the initial sextet into two nonequivalent six-line patterns in the recorded spectra. The values of the modulus $B_{\text {eff }, 1,2}$ given in Table II correspond to the outer and, respectively, to the inner sextet. The linewidth of the sextets is practically the same as for the zero-field spectra; i.e., there is no observable broadening. Moreover, both values $B_{\text {eff }, 1,2}$ increase with the applied field (Table II). This feature is of paramount importance and is one of the highlights of this paper, since it is direct evidence that the main contribution to $B_{\text {int }}$ is of orbital origin instead of Fermi's contact term, which is negative, as we shall argue in the next section.

To show this point better, in Fig. 4 the initial $B_{\text {int }}\left(=B_{\text {eff }}\right.$, for $B_{a p p l}=0$ ) and the $B_{e f f, i}$ (within the error bars) of the two sextets are plotted versus the applied field, together with the algebraic sum of the $B_{\text {int }}(4.2 \mathrm{~K})$ plus $B_{a p p l}$ (plotted as a dashed line), for the sake of comparison. At the $B_{a p p l}=5 \mathrm{~T}$, the two values are almost equally separated from the dashed line, but at $10 \mathrm{~T}$ there is a tendency of $B_{e f f, 1}$ vector to approach colinearity with $B_{a p p l}$. On the other hand, the $B_{\text {eff }, 2}$ values show, within experimental error, an almost linear dependence with the external field. It is evident by inspection of Fig. 4 that the $B_{e f f, 1}$ cannot be accounted for by simply the addition of $B_{a p p l}$ to $B_{\text {int }}$; therefore, there is an extra component of intrinsic origin induced by the field. The $\varphi$ angle, mentioned above, varied in the range between $55.4^{\circ}$ and

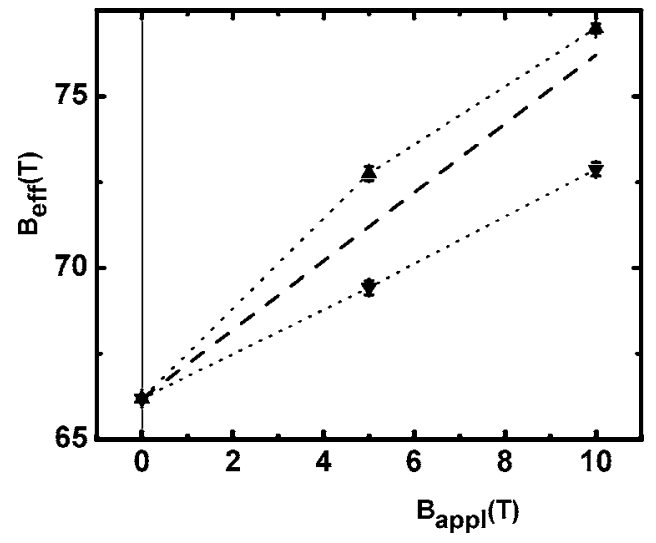

FIG. 4. Effective hyperfine field values of the in-field sextets; ( $\Delta) B_{\text {eff }, 1},(\boldsymbol{\nabla}) B_{\text {eff }, 2}$. The dashed line shows the algebraic sum of $B_{\text {int }}+B_{\text {appl }}$, i.e., for fully collinear arrangement, for comparison purposes.

$58.6^{\circ}$ (Table II), and is noticeably different from the value obtained for the measurements without applied field, proving that the average angle between the EFG axis and the easy magnetization direction (EMD) has also changed. Indeed, when applying a magnetic field, the magnetic interaction can modify the direction of the effective hyperfine field at the nucleus. The relative intensity of the $\Delta m=0$ lines of the sextet (number 2 and 5) with respect to the $\Delta m=1$ lines yields information on the angle $\theta$ between $B_{\text {eff }}$ and $\gamma$ rays. In the case when the quadrupole interaction is treated as a small perturbation and the absorber thickness tends to zero, for ${ }^{57} \mathrm{Fe}$, the relative intensities of the sextet lines, the outer, middle, and inner pairs of the sextet pattern, are given by

$$
3\left(1+\cos ^{2} \theta\right): 4 \sin ^{2} \theta:\left(1+\cos ^{2} \theta\right) .
$$

In many cases of measurements on polycrystalline samples or powders, due to a far from vanishing absorber thickness, texture, and competitive electronic mechanisms, the ratios show smaller values than the theoretical ones, calculated from Clebsch-Gordan coefficients. This is the case of the measurements at very low temperatures. In the absence of external applied field $\left(B_{a p p l}=0\right)$, a middle $(\Delta m=0)$ to inner line area ratio (relative intensities) of 1.72 (instead of the theoretical value of 2) was found. In the fitting procedure of the spectra measured under applied field, the ratio for the $\Delta m=0$ lines of each of the two sextets evolves independently. For the smaller $B_{\text {eff, } 2}$ fields, the following ratios for $\Delta m=0$ lines are obtained: 1.24 at $5 \mathrm{~T}$ and 1.03 at $10 \mathrm{~T}$, while for $B_{\text {eff, } 1}$ quite lower values are obtained, 0.54 and 0.23 at 5 and, respectively, $10 \mathrm{~T}$, but none of them vanishes. These features are directly reflected in the line intensities of the individual sextets as presented in Fig. 3. This is in agreement as well with Fig. 4 , where $B_{\text {eff }, 1}$ tends to align in the direction of the applied field and the incident $\gamma$ ray, while the $B_{\text {eff }, 2}$ shows an almost linear increase. The iron site corresponding to the higher $B_{e f f, 1}$ represents roughly $35 \%$ of iron population in $\alpha$-FePc both in fields of $5 \mathrm{~T}$ and, respectively, $10 \mathrm{~T}$.

It is not possible to establish very reliably the value of the angle between the EMD and the incident gamma ray in the 


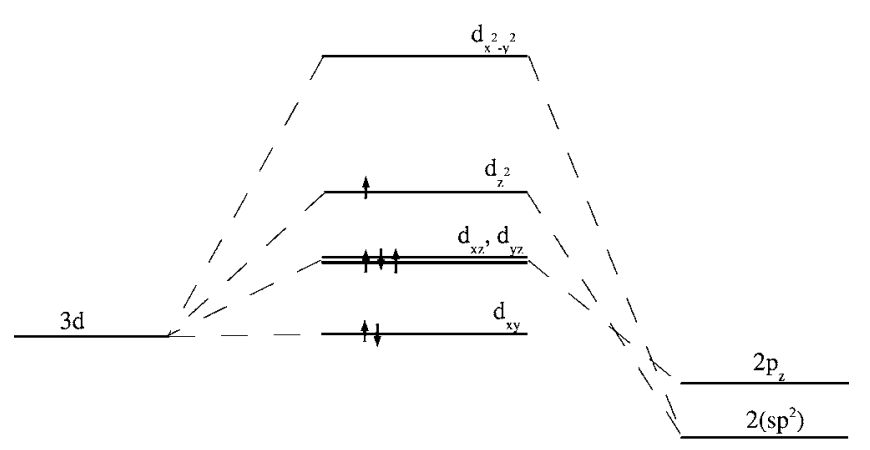

Fe orbitals molecular orbitals $\quad \mathrm{N}$ orbitals

FIG. 5. Molecular orbital energy level scheme for $\alpha$-FePc.

present case, due to the above-mentioned reasons. However, from the clearly decreasing trend of relative ratio between middle and inner line area for both sextets, one may conclude that the effective fields tend, as physically expected, to orient themselves closer to the incident ray direction, i.e., parallel to the direction of the applied field and out of the sample plane. This feature, in a parallel way, is also suggested by the variation of the $\varphi$ angle between the EFG axis and the EMD. It is evident that the two sublattices rotate differently under applied field; this peculiar behavior will be stressed during the discussion.

\section{DISCUSSION}

\section{A. Low-temperature Mössbauer data}

\section{Zero field}

It is useful to emphasize again that the $\alpha$-FePc is a coordination compound, where the nominally divalent iron is bonded with two out of four nitrogen atoms in the phthalocyanine plane, exhibiting an intermediate spin state $S=1$. The bond is time-shared between the two pairs of opposite nitrogen, leading to a strong orbital degeneracy. The mentioned $D_{4 h}$ symmetry could give rise to various configurations, but the interpretation of both magnetic and Mössbauer data points to the occupation scheme $3 d^{6}=d^{2}{ }_{x y}\left(d_{x z}, d_{y z}\right)^{3} d_{z^{2}}$, which was first proposed by Dale et al. ${ }^{27}$ and later confirmed by accurate X-ray diffraction data of Coppens et al. ${ }^{21}$

A simple calculation provides information on the expected ground state within the above-mentioned configuration, in the presence of the two highly orbitally degenerate levels $d_{x z}$ and $d_{y z}$. The so-called strong ligand field approach will be considered, ${ }^{31}$ whereby the zeroth-order wave functions of $\mathrm{Fe}$ (II) are essentially products of single-particle wave functions - the occupied eigenstates of the ligand field of the symmetry $D_{4 h}$, see Fig. 5, whereas the eigenenergies are assumed to include corrections of first order in the electron-electron interaction.

The nonelectrostatic nature of the ligand field is emphasized here. In order to overcome the intra-atomic Hund's exchange, which favors double occupation of the ground orbital and single occupation of all the excited ones, the top level $d_{x^{2}-y^{2}}$ must be situated at least $4(B+C) \approx 2.5 \mathrm{eV}$ higher than the $e_{g}\left(d_{x z}, d_{y z}\right)$ doublet (using the Racah parameters $B$ and $C$ for $\mathrm{Fe}$ (II) taken from Table 2.11 of Ref. 32). The electrostatic contribution to the ligand is readily demonstrated to be too small to account for these splittings, and therefore irrelevant.

To prove this point, let us present the electrostatic potential around $\mathrm{Fe}(\mathrm{II})$ as the effect of four point charges, $e$, situated on the neighboring nitrogen atoms (to make the point clearer, we deliberately exaggerate the charge value). The resulting splittings would be of the order of $e^{2}\left\langle r^{2}\right\rangle_{3 d} / d^{3}$ $\approx 0.8 \mathrm{eV}$ (by way of second-order ligand field) and $e^{2}\left\langle r^{4}\right\rangle_{3 d} / d^{5} \approx 0.02 \mathrm{eV}$ (fourth-order 1igand field), which is too little. The expectation values $\left\langle r^{2}\right\rangle_{3 d}=1.393 a_{0}^{2}$ and $\left\langle r^{4}\right\rangle_{3 d}$ $=4.496 a_{0}^{4}$ can be found in Table 7.6 of Ref. 31 and the ironnitrogen interatomic distance $d=1.927 \AA$ is from Ref. 20. In reality the charge on nitrogen is only a small fraction of the electron charge and the predicted electrostatic splittings should be scaled down proportionately.

Hereafter the electrostatic component of the ligand field is neglected and the splitting of the $3 d$ levels is ascribed entirely to hybridization of the $3 d$ orbitals with the orbitals of the neighboring nitrogen atoms. The latter come in two shapes, as $s p^{2}$ hybrids and as pure $p_{z}$ orbitals. ${ }^{33}$ Both are initially lower in energy than the unsplit $3 d$ level of iron, the $s p^{2}$ hybrids being some $4 \mathrm{eV}$ below the $p_{z}$ (as judged by the Hartree-Fock energies $\varepsilon_{s}$ and $\varepsilon_{p}$ from the solid state table of Ref. 34). The petals of the $s p^{2}$ hybrids stretch out towards the iron atom and form strong $\sigma$ bonds with both $d_{z}$ and $d_{x^{2}-y^{2}}$ iron orbitals. As a result, the hybridized antibonding $d_{z}$ and $d_{x^{2}-y^{2}}$ type states are pushed high up in energy. To second order in perturbation theory, the elevations of $d_{x^{2}-y^{2}}$ and $d_{z^{2}}$ above the original $3 d$ level are related as the squares of the corresponding Slater-Koster matrix elements (see Table 15-2 of Ref. 34), i.e., as 3:1. The $e_{g}$ doublet orbitals, $d_{x z}$ and $d_{y z}$, form weaker $\pi$ bonds with the $2 p_{z}$ orbitals of nitrogen and are pushed up only slightly. Finally, the $d_{x y}$ state is practically nonbonding, since it can only form very weak bonds with those $s p^{2}$ hybrids whose petals are directed towards the so-called bridge nitrogen atoms, away from the iron atom. Thus, the $d_{x y}$ orbital remains at the initial $3 d$ level.

As one can appreciate, the original level occupation scheme of Dale et al. ${ }^{14}$ finds a natural explanation in terms of the covalent chemical bond. One peculiarity of the ground configuration is that it is orbitally twice degenerate. Indeed, the spin-down electron on the $e_{g}$ level in Fig. 5 can be understood to occupy either the $d_{x z}$ or the $d_{y z}$ component of the doublet. The distinction between the two corresponding states of the $\mathrm{Fe}$ (II) ion is purely orbital since no change of the spin state of the system is involved. In addition, there is a fourfold degeneracy with respect to spin, as implied by the two unpaired electrons (or more conveniently, two holes, one on $e_{g}-\left(d_{x z}, d_{y z}\right)$, the other one on $\left.a_{1 g}-d_{z^{2}}\right)$. Thus, the total degeneracy of the ligand-field ground configuration is 8 .

The spin degeneracy is partially lifted by the electronelectron (hole-hole) interaction, which splits the ground configuration into a spin triplet $(S=1)$ and a spin singlet $(S=0)$, the latter being $2 C \sim 1 \mathrm{eV}$ higher in energy, both twice orbitally degenerate. (The time-even Coulomb interaction leaves 
unsplit the two Kramers conjugate orbital states). Thus, in the absence of spin-orbit coupling the ground state of $\mathrm{Fe}$ (II) in FePc is sixfold degenerate (a twice orbitally degenerate spin triplet). Its six components can be conveniently presented as two-hole states $\left|M_{L} M_{S}\right\rangle$, labeled with the magnetic $\left(M_{L}= \pm 1\right)$ and spin $\left(M_{S}=0, \pm 1\right)$ quantum numbers. For example,

$$
\begin{aligned}
| \pm 1,1\rangle & =\left|e_{g}^{1}, m_{s}^{1}=1 / 2\right\rangle\left|a_{1 g}^{2}, m_{s}^{2}=1 / 2\right\rangle \\
& =\mp 2^{1 / 2}\left(d_{x z} \pm i d_{y z}\right) d_{z^{2}}|\uparrow \uparrow\rangle, \text { etc. }
\end{aligned}
$$

where the superscript corresponds to the hole number.

Note that these are eigenstates of $\hat{L}_{z}, \hat{S}_{z}$, and $\hat{S}^{2} \doteq 2$ but not to $\hat{L}^{2}$. For convenience the $e_{g}$ doublet orbitals have been chosen as complex combinations of $d_{x z}$ and $d_{y z}$, $\mp 2^{-1 / 2}\left(d_{x z} \pm i d_{y z}\right) \equiv|l m\rangle, l=2, m= \pm 1$; this choice will ensure that the spin-orbit Hamiltonian (5) is diagonal. Strictly speaking, the orbital parts of the wave functions (1) should be antisymmetrical with respect to permutation of the holes. However, we shall not do it here, since we only intend considering such perturbing operators that act on each hole separately. The sixfold degeneracy of the group of states (4) is further partially lifted by the spin-orbit interaction. It is easy to see that the spin-orbit Hamiltonian,

$$
\hat{H}_{\mathrm{so}}=-\zeta\left(\hat{l}_{1} \cdot \hat{s}_{1}+\hat{l}_{2} \cdot \hat{s}_{2}\right)
$$

is diagonal in the $\left|M_{L} M_{S}\right\rangle$ representation. The minus sign reflects the fact that the operator (5) acts on holes rather than electrons, $\zeta$ is the usual one-electron spin-orbit coupling constant, $\zeta=410 \mathrm{~cm}^{-1}$, Table 2.11 of Ref. 32 . The result is the following three doublet states:

$$
\begin{gathered}
| \pm 1, \pm 1\rangle \text { ground, or " parallel," at } E_{0}=-\zeta / 2, \\
| \pm 1,0\rangle 1 \text { st excited “perpendicular" at } E_{1}=0, \\
| \pm 1, \mp 1\rangle 2 \text { nd excited “ antiparallel” at } E_{2}=\zeta / 2 .
\end{gathered}
$$

At temperatures below $\sim 100 \mathrm{~K}$ the magnetic properties of the system are determined exclusively by the ground doublet, so we turn our attention to it. Its most salient features are the following:

(i) The orbital moment is not quenched due to the orbital degeneracy-a hole occupying an orbital doublet, $\left\langle\hat{L}_{z}\right\rangle= \pm 1$.

(ii) The magnetic moment has an extremely anisotropic (Ising) character, $\left\langle 2 \hat{S}_{z}+\hat{L}_{z}\right\rangle= \pm 3$, whereas $\left\langle 2 \hat{S}_{x, y}+\hat{L}_{x, y}\right\rangle=0$. The fact that magnetic dipole transitions are not permitted between the components of the ground doublet may make thermal equilibrium difficult to reach. This could be the cause of the blocking phenomena observed at low temperatures. ${ }^{7}$

(iii) To first order, low-symmetry distortions of the ligand field are unable to split the ground doublet. Since it is not a true Kramers doublet, it will be split eventually by the combined action of the spin-orbit coupling and low-symmetry ligand field in higher orders of perturbation theory. If the deviation from the ideal $D_{4 h}$ is described by a small dimensionless parameter $\varepsilon$ (by analogy with elastic strain in the theory of magnetostriction ${ }^{35}$ ) the splitting of the ground doublet will be $\sim \varepsilon \zeta$, i.e., rather small and will not stand in the way of magnetic ordering.

This scheme allows us to interpret the large orbital moment contribution to $B_{\text {int }}$. In a magnetically ordered compound it contains three main contributions,

$$
B_{\text {int }}=B_{F}+B_{L}+B_{D} .
$$

Here $B_{F}$ is the Fermi contact field arising from the polarization of the core electrons via the exchange interactions with the valence electrons. Generally this term is proportional to the net spin density at the nucleus. $B_{L}$ is the magnetic field produced at the same nucleus by the orbital motion of the electrons from a partially filled shell. $B_{D}$ is the dipolar field due to the spin magnetization of the valence shell. It is largely agreed that in most cases the main contribution to $B_{\text {int }}$ comes from $B_{F}$, specifically in the case of ions with a half-filled shell [for example, $\mathrm{Fe}(\mathrm{III})$ ]; however, as it is shown below, this is not the case in the square planar $S=1 \mathrm{Fe}(\mathrm{II})$. The detailed expressions of the three components could be written, for instance in the way presented in Ref. 36.

The Fermi contact field contribution is

$$
B_{F}=\frac{8}{3} \pi \mu_{B}\left[\rho_{\uparrow}(0)-\rho_{\downarrow}(0)\right],
$$

where $\rho(0)$ is the electron density at Fe nucleus, with spin up and, respectively, spin down.

The orbital contribution provides a field:

$$
B_{L}=-2 \mu_{B}\left\langle r^{-3}\right\rangle_{3 d}\left\langle L_{z}\right\rangle .
$$

Finally, the dipolar field contribution is

$$
B_{D}=2 \mu_{B} q_{3 d}\left\langle S_{z}\right\rangle .
$$

Considering the electronic configuration of $3 d$ levels, $d_{x y}^{2}\left(d_{x z}, d_{y z}\right)^{3} d_{z^{2}}^{1} d_{x^{2}-y^{2}}^{0}$, the factor that appears in Eq. (11), is $q_{3 d}=-\frac{2}{7}\left\langle r^{-3}\right\rangle_{3 d}$, as derived from Ref. 37 .

According to the theoretical prediction, the ground state presents the values $\left\langle L_{z}\right\rangle=\left\langle S_{z}\right\rangle=-1$. Therefore the two terms could be also written as

$$
B_{L}=2 \mu_{B}\left\langle r^{-3}\right\rangle_{3 d} \text { and } B_{D}=\frac{4}{7} \mu_{B}\left\langle r^{-3}\right\rangle_{3 d} .
$$

In the above expressions, most of the factors are known physical constants, while for $\left\langle r^{-3}\right\rangle_{3 d}$ and $B_{F}$ terms some estimations are possible. The evaluations will take into consideration various calculated $\left\langle r^{-3}\right\rangle_{3 d}$ values and the reported $B_{F}$ data for different configurations of iron, both for tri- and bivalent iron in spin-only states.

The classical Hartree-Fock calculations provide the following values for $\left\langle r^{-3}\right\rangle_{3 d}$ : $\mathrm{Fe}(\mathrm{IV})(S=2) 6.33$ a.u., $\mathrm{Fe}(\mathrm{III})$ $(S=5 / 2) 5.72$ a.u., and Fe(II) $(S=2) 5.08$ a.u. (Table 7.6 in Ref. 31). From this sequence one could expect a value even lower than 5.0 a.u. for the $S=1$ intermediate spin state of the bivalent iron (tentatively estimated between 4.50 and 4.90 a.u.). Indeed in their paper, Watson and Freeman ${ }^{38}$ discussed the possible reduction of the $\left\langle r^{-3}\right\rangle_{3 d}$ electric quadru- 
pole case to a value of 4.93 a.u. and to an even smaller value of 4.59 a.u. for the orbital case and down to 4.55 a.u. for dipolar case. All estimations are referring to the classical free ion configuration, that is, the highest ionic state. It is observed, from reasons related to the strength of the electronic interactions with various anions, that the mentioned $\left\langle r^{-3}\right\rangle_{3 d}$ values are noticeably decreasing in the direction from right to left in the periodic table, i.e., Fe- $X$ bonds are weaker in the trend from $X=\mathrm{F}, \mathrm{O}, \mathrm{N}$ to $\mathrm{C}$.

On the other hand, the $B_{F}$ contribution strongly depends on the strength of ionic bond and dimensionality. ${ }^{30}$ Indeed the largest $B_{F}$ was found for $\mathrm{FeF}_{3}\left(B_{h f}=62.2 \mathrm{~T}\right)$, a 3D compound, while a reduction of $14 \%$ occurs for the twodimensional (2D) one, $\mathrm{RbFeF}_{4}$, and further decrease to $34 \%$ for the one-dimensional (1D) structure, in $\mathrm{K}_{2} \mathrm{FeF}_{5}$.

It is accepted use to evaluate the ratio of $B_{F}$ with respect to the spin value as a method of assessment of the $B_{F}$ contribution in measured systems and extrapolate it to cases where it cannot be directly measured. It has to be mentioned that in the case of high-spin $\mathrm{Fe}$ (III) with a half-filled $\left(3 d^{5}\right)$ shell, the only contribution to effective field at the nucleus comes from Fermi-contact term $B_{F}$; therefore, it is a convenient reference. Taking the reported value of $62.2 \mathrm{~T}$ for the five $d$ electrons, in the most ionic configuration, one obtains $12.44 \mathrm{~T}$ per $S=1 / 2$ value. However, for Fe(III) oxides, a lower value of $11 \mathrm{~T}$ per $S=1 / 2$ is mentioned. ${ }^{36}$ When the proportionality is referred to the ferrous compound the experimental data provide the highest value $B_{F}$, of $32.9 \mathrm{~T}$ for $\mathrm{Fe}(\mathrm{II})$ in $\mathrm{FeF}_{2}$ (Ref. 39), i.e., $8.225 \mathrm{~T}$ per one-half spin.

But in the case of weak bonds, far from highly ionic structure, in the metal-metalloids or coordination compounds, much lower values are observed for $B_{F}$. One example is ferrous oxalate $(S=2)$ showing $B_{F}=15.0 \mathrm{~T} .{ }^{40}$ The derived halfspin value is $3.75 \mathrm{~T}$. Unfortunately, there is no report, to the best of our knowledge, of an iron spin-only ordered system, with $S=1$ spin state, to compare the Fermi contact value.

Let's consider the two most obvious options to evaluate $B_{\text {int }}$ (in the limits of two decimals):

(a) taking only the largest values: $\left\langle r^{-3}\right\rangle_{3 d}=5.08 a_{0}^{-3}$ the relations (10) yield a $B_{L}=63.55 \mathrm{~T}$ and, respectively, $B_{D}=18.16 \mathrm{~T}$, and considering the largest $B_{F}=-16.45 \mathrm{~T}$, derived from Ref. 38, one obtains $B_{\text {int }}=65.26 \mathrm{~T}$.

(b) taking only the smallest values from Ref. 37, providing $\left\langle r^{-3}\right\rangle_{L}=4.59 a_{0}^{-3}$, that gives rise to $B_{L}=57.42 \mathrm{~T}$ and with $\left\langle r^{-3}\right\rangle_{D}=4.55 a_{0}^{-3}$ yields $B_{D}=16.26 \mathrm{~T}$. Taking the smallest $B_{F}=-7.5 \mathrm{~T}$ (Ref. 39), the resulting sum is $B_{\text {int }}=66.18 \mathrm{~T}$.

The values are very close to the $\alpha-\mathrm{FePc}$ sample $\left(B_{i n t}\right.$ $=66.2 \mathrm{~T})$ but noticeably larger than the one $\left(B_{\text {int }}=62.1 \mathrm{~T}\right)$ with a similar local Fe surrounding $\left(\alpha\right.$-FeOETAP). ${ }^{29}$

It may be concluded that in the case of $\alpha$-FePc [and $\alpha$ -FeOETAP (Ref. 41) as well], the large orbital moment gives rise to a positive $B_{L}$ and the dominant ferromagnetic interaction produces a large, also positive, $B_{D}$. Both positive terms, far exceeding the negative Fermi contact one, favor the appearance of the highest internal field ever observed at the ${ }^{57} \mathrm{Fe}$ nucleus in bivalent iron intermediate spin configuration. For the sake of completeness, we collected common magnetic and Mössbauer data on the square planar compounds with $S=1$ intermediate spin (see Table III). The paramagnetic
TABLE III. Effective magnetic moments, hyperfine fields and quadrupole splittings for the $\alpha$ and $\beta$ polymorphs of FeOETAP and FePc. The $\Delta E_{Q}$ given are the room temperature values, whereas the hyperfine fields were determined at low temperatures: $T=1.3 \mathrm{~K}$ $(\alpha$-FePc $)$ and $1.08 \mathrm{~K}(\alpha$-FeOETAP $)$.

\begin{tabular}{lccc}
\hline \hline \multicolumn{1}{c}{ Compound } & $\mu_{\text {eff }}\left(\mu_{\mathrm{B}}\right)$ & $\begin{array}{c}B_{\text {int }} \\
(\mathrm{T})\end{array}$ & $\begin{array}{c}\Delta E_{Q} \\
(\mathrm{~mm} / \mathrm{sec})\end{array}$ \\
\hline$\alpha$-FePc & $3.6^{\mathrm{a}}$ & $66.2^{\mathrm{b}}$ & $2.52^{\mathrm{b}}$ \\
$\beta$-FePc & $3.7^{\mathrm{c}}$ & - & $2.57^{\mathrm{d}}$ \\
$\alpha$-FeOETAP & $3.8^{\mathrm{e}}$ & $62.4^{\mathrm{f}}$ & $2.86^{\mathrm{e}}$ \\
$\beta$-FeOETAP & $3.9^{\mathrm{f}}$ & - & $3.06^{\mathrm{f}}$ \\
\hline \hline
\end{tabular}

${ }^{\mathrm{a}}$ Reference 7.

bThis work.

${ }^{\mathrm{c}}$ Reference 14.

${ }^{\mathrm{d}}$ Reference 17.

${ }^{\mathrm{e}}$ Reference 41.

${ }^{f}$ Reference 29.

$\beta$ phases have quite comparable $\Delta E_{Q}$ and $\mu_{\text {eff }}$, though slightly higher for the FeOETAP compounds, and the $\alpha$ phases have similar $B_{\text {int }}$ but with a maximum value in the $\alpha$-FePc case. We discuss the reason for this difference below.

The larger value of the two energetic Mössbauer parameters $\left(B_{\text {int }}\right.$ and $\left.\Delta E_{Q}\right)$ for the FePc respect to the FeOETAP compounds, which have an identical first coordination sphere around $\mathrm{Fe}(\mathrm{II})$, may be naturally related to the ligands situated in the external rings structure. Such effects, even influenced by valence ligands far positioned from the central iron, are often observed in various coordination compounds, including frustrated spin systems. ${ }^{42}$ In contrast, the powder $\mu_{\text {eff }}$ are lower in the powder FePc compounds. To explain this different trend, it must be recalled that $B_{L}$ is proportional to the expectation value of the orbital moment along the easy direction $\left\langle\hat{L}_{z}\right\rangle \approx-1$, while $\mu_{\text {eff }}$ depends on $\left\langle\hat{L}_{x y}^{2}\right\rangle$ and $\left\langle\hat{L}_{z}^{2}\right\rangle$. Therefore, this implies that in these anistropic compounds the $\alpha$-FePc has a larger $\left\langle\hat{L}_{z}\right\rangle$ and the FeOETAP have higher transverse orbital moments $\left\langle\hat{L}_{x y}^{2}\right\rangle$.

Although not directly related with FePc compounds, the planar three-coordinated high-spin $\mathrm{Fe}(\mathrm{II})$ complexes $L \mathrm{Fe}^{\mathrm{II}} X$, with $L=b$-diketiminate and $X=\mathrm{Cl}^{-}, \mathrm{CH}^{3-}, \mathrm{NHTol}^{-}, \mathrm{NhtBu}^{-}$, also show large values of the orbital moment, or order $\left|L_{z}\right|$ $=1$, as deduced from Mössbauer and magnetic measurements. The origin of the large $L_{z}$ was also traced back to the existence of orbitally degenerate levels in the Fe electronic configuration. ${ }^{43}$

\section{In field}

The observed linewidths of the in-field spectra are close to those of the $4.2 \mathrm{~K}$ spectrum without applied field. This is in agreement with a similar observation of rather sharp lines for bulk barium ferrite data in applied field reported by Pankhurst et al. ${ }^{44-46}$ Small linewidths were also reported in experiments on hematite, ${ }^{47}$ magnetoferritin,${ }^{48}$ and neutronirradiated hematite ${ }^{49}$ under applied field. On the contrary in disordered systems of maghemite, ${ }^{50}$ glued goethite, ${ }^{51}$ and substituted barium ferrite, ${ }^{45}$ broadened lines appeared. 


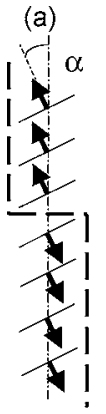

(b)

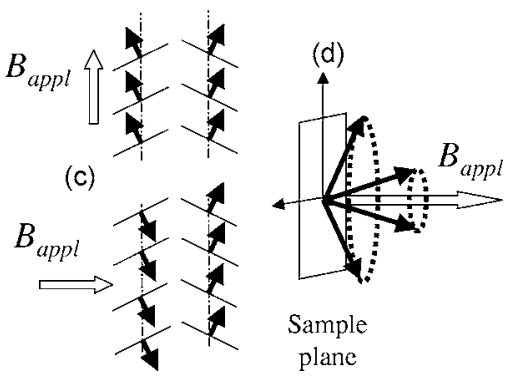

FIG. 6. Schematic representation of the $\alpha$-FePc magnetic chains and domains. The magnetic moment canting angle to the $b$ axis is $\alpha$. (a) Ferromagnetic chain with two domains separated by a 1800 wall. (b) Two sublattice magnetic domain oriented by an external field applied parallel to the $b$ axis, or with dominant projection along this axis. (c) The field applied perpendicular to the $b$ axis, or with dominant projection along this axis. (d) Cones of average orientation of the spins pertaining to the two types of domains under an applied field perpendicular to the sample plane. The narrow cone concerns $B_{e f f, 1}$ and the wide cone concerns $B_{e f f, 2}$.

The mean field model coupled to simulations of the Mössbauer spectra for a given distribution of orientation probabilities $^{44,45}$ provides an accurate fit of spectra and reliable parameters for polycrystalline powders or disordered systems. For anisotropic systems this model predicts a splitting of the lines due to the existence of different magnetic sublattices, but also that the line shapes are broader with the increasing angular distribution due to increasing degree of disorder, spin anisotropy, and applied fields. The absence of broadening in the in-field spectra leads us to rule out that we encounter a simple case of random distribution of highly anisotropic spins. Instead, it is a clear indication that the applied field reorients the magnetic moments in a rather definite configuration.

$\mathrm{In}^{7}$ it was demonstrated that $\alpha$-FePc follows an Ising-like behavior of magnetic chains of $S=1$ spins with ferromagnetic intra-chain exchange and anti-ferromagnetic inter-chain coupling. There was also evidence of the existence of two $\mathrm{Fe}$ sub-lattices with a canting angle respect to the $b$-axis, however, it was not possible to ascertain this feature with neutron diffraction because of the strong incoherent background due to the abundant $\mathrm{H}$ atoms. Such canted structure gives rise to a division of the crystal in ferromagnetic domains [Fig. 6(a)] of canted moments with net magnetization along the magnetically degenerate directions, similar to that found in MnPc. ${ }^{52}$ Under a strong external field, as is the case in the Mössbauer experiments, the domains with net moment close to the field direction grow at the expense of the other domains, till all magnetic moments have a positive projection on the direction of the field $\left(\boldsymbol{B}_{\text {appl }}\right)$ [Figs. 6(b) and 6(c)]. In the present compound when the rodlike grains are placed on a flat surface and are tightly pressed, as is the Mössbauer sample holder, the rods orient preferentially with their long axis parallel to the mentioned surface and distributed at random in a plane (Section II). Therefore, one may expect a rather strong texture effect which, combined with $B_{\text {appl }}$ favors those domains with a non-zero net moment perpendicular to the $b$ axis to be aligned in the field direction [Fig. 6(d)].
On the other hand, because of the random orientation of the rods parallel to the sample holder plane the domain net moments are distributed onto a cone with positive projection in the field direction.

Though we have visually observed the planar random distribution of the rods on the supporting plane, there is a large fraction of them with their $b$-axis out of this plane. Those grains at an angle smaller than the $\alpha$ canting angle with respect to the applied field direction would have magnetic domains with net moment perpendicular to the previous set of grains [Fig. 6(d)]. Their spins would be distributed in a second cone around the field direction with a cone- angle complementary to the first one. It is plausible to expect, for the above reasons, the existence of two subsets of Fe spins with different averaged $B_{e f f}$, that is, the spins within the narrow cone concern $B_{e f f, 1}$, and those in the wider cone concern $B_{e f f, 2}$ [see Fig. 6(d)].

From the Mössbauer point of view, the two sites are completely identical in the absence of any external field. The in-field Mössbauer results point definitely to extremely large values of both $B_{L}$ and $B_{D}$. It is clear that both sextets show an increased $B_{\text {eff }}$, with higher applied field, confirming the $\mathrm{Fe}-\mathrm{Fe}$ ferromagnetic dominant interaction already derived from the magnetic data. ${ }^{7}$ The $B_{e f f, 1}$ showed larger (1-2.5\%) values than algebraic sum of $B_{i n t}$ and $B_{a p p l}$, the difference (Fig. 4) diminishing with increasing external field while $B_{\text {eff }, 2}$ presents smaller values than the algebraic sum and shows larger differences (Table II and Fig. 4) to the mentioned sum with increasing field. On the other side, the differences between the two $B_{e f f}$ values are also increasing with applied field.

From the experimental geometry, it is possible ${ }^{53}$ to determine the average angle of the cone,

$$
\vartheta=\arccos \left[\left(B_{\text {eff }, 2}^{2}-B_{\text {appl }}^{2}-B_{\text {int }}^{2}\right) /\left(2 B_{\text {appl }} B_{\text {int }}\right)\right],
$$

amounting to $\vartheta=53.50^{\circ}$. There is no way to apply the same formula for $B_{e f f, 1}$ since $B_{e f f, 1}$ is somewhat larger than the sum of $B_{\text {int }}$ and $B_{\text {appl }}$ if collinear. This implies that there is an extra contribution induced by the applied field, besides that of orienting the moments in the narrow cone. The extra contribution could arise from the orbital contribution via a spinorbit interaction of the highest energy orbitals $d_{y z}$ and $d_{z^{2}}$ pointing directly to an increase of the $\left\langle L_{z}\right\rangle$ value.

\section{B. Magnetic measurements}

The ac magnetic susceptibility was measured on the same batch of $\alpha$-FePc on which the Mössbauer study has been performed (Fig. 7). The real component $\chi^{\prime}(T)$ data agree with the measurements shown in Ref. 7. They had been interpreted in terms of an Ising $S=1$ magnetic chain model with ferromagnetic intrachain interaction $J_{1} / k_{\mathrm{B}}=25.7 \mathrm{~K}$, $g=2$, and a strong crystal field with $D / k_{\mathrm{B}}=53.2 \mathrm{~K}$. The net effect of these interactions is to produce a ground state doublet. In the present paper, the prediction for the ground state is also a doublet, but differs from the previous in the correct consideration of $M_{L}$. Therefore, the Ising model with the effective $S=1 / 2$ has been applied to interpret the magnetic low-temperature data. 


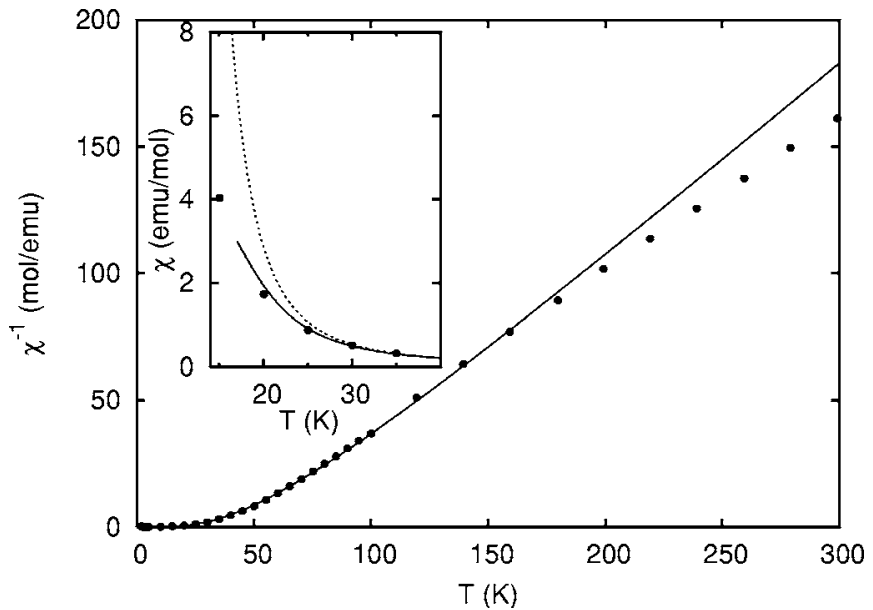

FIG. 7. Temperature dependence of the magnetic susceptibility of $\alpha$-FePc (inset) and of its inverse (main plot). The data points are from Ref. 7. The solid curves were calculated within the $S=1 / 2$ Ising model on a tetragonal lattice with $J_{\mathrm{z}} / k_{\mathrm{B}}=76 \mathrm{~K}$, $J_{\mathrm{xy}} / k_{\mathrm{B}}=-0.1 \mathrm{~K}, g_{\|}=6.4$. The dotted curve in the inset is the prediction for an isolated $S=1 / 2$ Ising chain with the same parameters.

The continuous line in Fig. 7 is a fit to the data between 20 and $150 \mathrm{~K}$ obtained with the Ising model on an anisotropic tetragonal model, where the ratio of exchange constants $J_{\mathrm{xy}} / J_{\mathrm{z}}=0$ corresponds to the chain model, with $z$ as the chain direction, $J_{\mathrm{xy}} / J_{\mathrm{z}}=1$ to the simple cubic model, and intermediate ratio values correspond to the presence of interchain interactions. ${ }^{54}$ The powder susceptibility has been calculated as $\chi_{\mathrm{p}}=1 / 3 \chi_{\|}$, since $g_{\perp}=0$. The exchange integral between nearest neighbors in one direction is positive and large, $J_{\mathrm{Z}} / k_{\mathrm{B}}=76 \pm 2 \mathrm{~K}$ (or $J_{\mathrm{z}} / k_{\mathrm{B}}=38 \mathrm{~K}$ in the notation of Ref. 54 , which differs from the standard convention by a factor of $1 / 2)$. This represents the ferromagnetic interaction within the $\alpha$-FePc chains. The exchange integral between nearest neighbors in the other two directions is small and negative, $J_{x y} / k_{\mathrm{B}}=-0.1 \mathrm{~K}$, which represents the weak antiferromagnetic interchain interaction. The best-fit splitting factor is $g_{\|}=6.4$ (the model assumes $g_{\perp}=0$ ); hence, for randomly oriented powder $\mu_{\text {eff }}=\left(g_{\|} / 2\right) \mu_{\mathrm{B}}=3.2 \mu_{\mathrm{B}}$, in good agreement with the experimental value $3.6 \mu_{\mathrm{B}}$ (Table III). As one would expect, the $S=1 / 2$ Ising model deviates from the experimental data above the temperature corresponding to one-half of the gap separating the ground and first excited doublets, $\zeta / 4 k_{\mathrm{B}} \approx 150 \mathrm{~K}$. On the other hand, at low temperatures, the model successfully describes the data down to the lower convergence bound of the high-temperature series of Ref. 54 . The dotted line in the inset of Fig. 3 is the prediction for an isolated $\left(J_{x y}=0\right) S=1 / 2$ Ising chain with the same $J_{z}$ and $g_{\|}$ as above. Thus, despite the weakness of the interchain interaction, $J_{x y} / J_{z} \approx-1.3 \times 10^{-3}$, its effect is quite pronounced at $T \leqslant 20 \mathrm{~K}^{55}$

It was shown ${ }^{7}$ that the $\alpha$-FePc ac susceptibility presented a frequency dependence of the maximum in the real and complex components, which was explained as being caused by the gradual slowing down of the spin fluctuations with temperature due to the decrease of the density and the mobility of the walls, followed by nearly complete pinning below $5 \mathrm{~K}$. It was argued that domain wall motion, or kinks

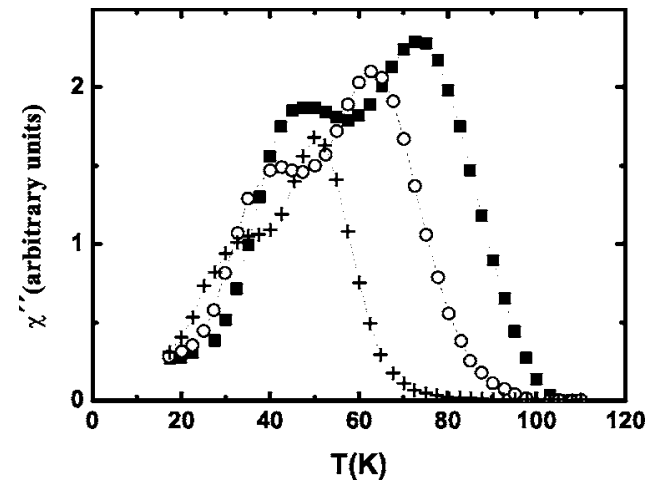

FIG. 8. Out-of-phase component of the ac susceptibility of powder $\alpha$-FePc in a bias external field of $0.08 \mathrm{~T}$. (+) $f=1 \mathrm{~Hz},(\bigcirc)$ $f=90 \mathrm{~Hz}$ and $(\boldsymbol{\square}) f=1400 \mathrm{~Hz}$.

(solitons), may induce that relaxation behavior. In such ferromagnetic linear chains, one expects to find single kinks originating at the ends of the finite chains or at defects, and double kinks which are generated within the segment and each traveling in opposite sense, the activation energy of the latter being double that of the former. This model has explained satisfactorily the frequency dependent ac susceptibility of Ising $S=1 / 2$ ferromagnetic chains. ${ }^{56}$

The ac susceptibility was remeasured, this time in a bias fields up to $0.1 \mathrm{~T}$, as external symmetry breaking field, and was found that the previously observed maximum in the imaginary component $\chi^{\prime \prime}(\mathrm{T}, \omega)$ splits in two peaks, with maxima at higher $\left(T_{m 1}\right)$ and lower $\left(T_{m 2}\right)$ temperature (see Fig. 8). The values of $T_{m 1}$ and $T_{m 2}$ do not depend on the applied field at values higher than $0.01 \mathrm{~T}$.

From the Arrhenius law $\tau=\tau_{0} \exp \left(-E_{a} / k_{B} T\right)$, the expression $\ln \left(1 / \omega_{\text {exp }}\right)=\ln \left(\tau_{0}\right)+E_{a} / k_{B} T_{m}$ is derived for $\tau=1 / \omega_{\text {exp }}$. When applied to the $T_{m i}$ data, the activation energies of $E_{a 1}=72 \pm 1 \mathrm{~K}$ and $E_{a 2}=116 \pm 1 \mathrm{~K}$, with the same $\tau_{0}=2 \times 10^{-11} \mathrm{sec}$ for both processes, (see Fig. 2, inset) were found. The activation energy of the single-kink type of soliton is the creation energy of the $180^{\circ}$ domain wall in the $S$ $=1 / 2$ Ising ferromagnetic chain, $E_{a}=E_{s k}=4 J_{z} S^{2}=J_{z}$ where $J_{z}$ is the intrachain exchange interaction. For the pair-kink type the activation is $E_{a}=E_{p k}=2 E_{s k}$. Therefore, taking the value derived from the $\chi^{\prime}(T)$ fit the activation energies should be $E_{a 1}=76 \mathrm{~K}$ and $E_{a 2}=152 \mathrm{~K}$. The agreement with the $E_{a 1}$ experimental value is excellent, while that for $E_{a 2}$ is overestimated but acceptable in view of the approximations done. In conclusion, the soliton model explains qualitatively the experimentally observed magnetic relaxation under field, in the ac susceptibility of the $\alpha$-FePc.

As a corollary of the coupling model of Sec. IV A, the saturation magnetization is obtained. Thus, in a magnetic field applied along the Ising axis $z$ the saturation moment, $\mu_{z}=3 \mu_{\mathrm{B}}$. In a powder sample with perfectly random distribution of Ising axes, the low-temperature saturation value will be $\mu_{s}=\mu_{z} / 2=1.5 \mathrm{~B}$. Further growth of magnetization is expected to be slow, the slope being of the order of $\mu_{B}^{2} / \zeta \sim 10^{-3} \mu_{\mathrm{B}} /$ Tesla. This is in good agreement with the experimental result of $\mu_{s}=1.6 \mu_{\mathrm{B}}$ and slow increase with increasing field up to $20 \mathrm{~T}$ reported in Ref. 7. 


\section{Relaxation process}

The strong broadening of the Mössbauer spectra, in the temperature region 5 to $20 \mathrm{~K}$ (that has been described in Sec. III A), may be interpreted as due to spin dynamics within the ferromagnetic chains.

The same soliton relaxation model as proposed in the previous section may explain the relaxation behavior observed in the present Mössbauer spectra. Once created, the single kinks or kink pairs propagate along the chains. Because of their passage, the flip rate $\Gamma_{\omega}$ of the electron spins becomes proportional to the product $n_{s} v_{s}$ of wall density and wall average velocity. ${ }^{57}$ As a consequence, the electronic spins fluctuate and via the hyperfine interaction with the Fe nuclei the Mössbauer spectra are broadened when $\Gamma_{\omega}$ becomes close to the nuclear Larmor frequency $\omega_{L}$ value. The broadening disappears when either $\Gamma_{\omega} \gg \omega_{L}$ or $\Gamma_{\omega} \ll \omega_{L}$. The details about the wall type, whether Bloch or steplike, are not important. The spin-flopping time-dependent process, due to the passage of a $\pi$ soliton, is the mechanism that determines $\Gamma_{\omega}$. The excess broadening $\Delta \Gamma$ due to solitons of the Mössbauer linewidth with respect to the paramagnetic linewidth has been shown ${ }^{56}$ to be given by

$$
\Delta \Gamma \propto \Gamma_{\omega} /\left(\omega_{L}^{2}+\Gamma_{\omega}^{2}\right),
$$

where $\Gamma_{\omega}=4 n_{s} v_{s} / \sqrt{\pi}$ is the flipping rate of the electron spin. It has been shown that either for Ising or classical spin chains the flipping rate for the kink-wall motion is

$$
\Gamma_{\omega}=\Gamma_{0} \exp \left(-E_{a} / k_{B} T\right),
$$

where $E_{a} \cong E_{s}$ or $E_{a} \cong 2 E_{s}$, depending on the energy for the creation of single-or pair-kink soliton state. ${ }^{57}$ As a result, substituting (13) in (12), a function $\Delta \Gamma(T)$ is obtained, which is driven by the characteristic energy $E_{a}$, and shows a maximum at the temperature when the condition $\omega_{L}=\Gamma_{\omega}(T)$ is fulfilled (see Fig. 2).

The Larmor frequency is obtained from the expression

$$
\omega_{L} / 2 \pi=\left|g_{e}\right| \mu_{N} B_{h f} / \hbar=3.2 \times 10^{8} \mathrm{~Hz},
$$

where $g_{3 / 2}=-0.10$ is the ${ }^{57} \mathrm{Fe}$ excited state gyromagnetic factor, ${ }^{58}$ by substituting $B_{h f}=B_{\text {int }}=66.2 \mathrm{~T}$, as found in the previous section. We have fitted the available $\Delta \Gamma(T)$ data with this model (see Fig. 2) and obtained a value of $E_{a}=72 \pm 2 \mathrm{~K}$, and $\Gamma_{\mathrm{o}}=10^{12} \mathrm{rad} / \mathrm{sec}$ for the prefactor in $\mathrm{Eq}$. (12). The activation energy determined from the Mössbauer data is in excellent agreement with the value provided by the ac susceptibility for the single-kink process. On the contrary, the kink-pair process cannot be observed in the Mössbauer relaxation.

There is an extra linewidth broadening of $0.16 \mathrm{~mm} / \mathrm{sec}$ in the Mössbauer experimental spectra at low temperatures as compared to the high-temperature paramagnetic linewidth, which can be ascribed to the spin-wave contribution of sufficiently long chains to support their existence below the magnetic ordering temperature $11.8 \mathrm{~K}$.

From these experimental data, one may conclude that in $\alpha$-FePc the density of thermally excited domain walls or solitons along the chains determines the fluctuation rate. At high temperature, the density is very high and the fluctua- tions too fast to be observable; at intermediate temperatures, the fluctuation rate passes the frequency window imposed by the experiment, either ac susceptibility or Mössbauer, while at low temperatures the soliton density becomes too small and the walls become pinned by interchain interactions or defects in the chains.

\section{CONCLUSIONS}

The present Mössbauer investigation of $\alpha$-iron(II) phthalocyanine provides a very large and positive hyperfine field of $B_{h f}=66.2 \mathrm{~T}$ for a bivalent iron with spin $S=1$, stemming from the large and positive contributions of $B_{L}$ and $B_{D}$, which strongly exceed the negative $B_{F}$ one. It is demonstrated that the origin of these extraordinary contributions is of orbital origin. There is an electronic configuration $d_{x y}^{2}\left(d_{x z}, d_{y z}\right)^{3} d_{z^{2}}$ that gives rise to an orbitally degenerate ground state, and as a consequence an expectation value $\left\langle\hat{L}_{z}\right\rangle \approx-1$.

The in-field Mössbauer measurements directly confirmed the positive and very large contributions above stated, a result that is highlighted. The spin canting of the Fe moments in the magnetic chains previously proposed ${ }^{7}$ has been directly proven; however, the canting angle could not be determined unambiguously.

The values higher than the algebraic sum, $B_{\text {int }}+B_{\text {appl }}$, obtained for effective fields at the Fe nuclei, both at $B_{\text {appl }}=5$ and $10 \mathrm{~T}$, are interpreted in terms of an additional large orbital contribution to the effective field at the nucleus.

The magnetic susceptibility is interpreted in terms of an Ising effective $S=1 / 2$ model, since only the ground state doublet is populated at low temperatures, with a ferromagnetic intrachain interaction of $J_{z} / k_{\mathrm{B}}=76 \pm 2 \mathrm{~K}$. From the frequency dependence of the quadratic component of the ac susceptibility measurements, two spin dynamical processes are evidenced, with activation energies $E_{a 1}=72 \pm 1 \mathrm{~K}$ and, respectively, $E_{a 2}=116 \pm 1 \mathrm{~K}$. They are explained as due to excitation of single-kink and pair-kink solitons within the finite ferromagnetic chains.

The experimental linewidth values as a function of temperature point to a relaxation process with incipient blocking temperature around $12 \mathrm{~K}$ and with the linewidth value in the ordered region $(0.54 \mathrm{~mm} / \mathrm{sec}$ at $1.3 \mathrm{~K})$ higher than those observed in the paramagnetic region $(0.38 \mathrm{~mm} / \mathrm{sec}$ above $50 \mathrm{~K}$ ). The relaxation process in the range of 5-20 K, points again to a soliton contribution of single-kink excitation with activation energy of $72 \pm 1 \mathrm{~K}$.

The activation energies for single-kink excitations obtained by two different methods, ac susceptibility and Mössbauer spectroscopy, are in pretty good agreement, within the limits of errors. These experimental results are consistent with the predicted domain wall energy barrier for an $S=1 / 2$ chain of $E_{S}=J_{z} / k_{\mathrm{B}}=76 \pm 2 \mathrm{~K}$, if the exchange constant is derived from the real component of the susceptibility.

\section{ACKNOWLEDGMENTS}

This work has been supported by Project No. MAT05/ 1272 of the Spanish Ministry of Education and Science. M. D. Kuz'min acknowledges Grant No. SAB2000-0084. G. F. 
acknowledges the Royal Society Research Grant at the University of Liverpool and Grant No. SAB2003/0226 from Ministerio de Education, Cultura y Deporte of Spain. G. F. is also deeply grateful to Dr. Dominic Dickson for his kind support and encouragement during the visit in the laboratory of Liverpool University. The final part of this work was facilitated and supported by EU Network of Excellence MAGMANet.
*Present address: IFW Dresden, PF270116, 01171 Dresden, Germany.

${ }^{1}$ Phthalocyanines: Properties and Applications, edited by C. C. Leznoff and A. B. Lever (VCH Publishers, New York, 1996), Vol. 4.

${ }^{2}$ H. Eicher and A. Trautwein, J. Chem. Phys. 50, 2540 (1969).

${ }^{3}$ N. A. Bell, J. S. Brooks, S. D. Forder, J. K. Robinson, and S. C. Thorpe, Polyhedron 21, 115 (2002).

${ }^{4}$ G. Filoti, V. Kuncser, H. Franke, T. Kardinahl, and G. Manivannan, J. Radioanal. Nucl. Chem. 190, 315 (1995).

${ }^{5}$ V. Kuncser, G. Filoti, A. Avramescu, R. Poldarski, M. Biebricher and H. Franke, J. Alloys Compd. 257, 285 (1997).

${ }^{6}$ S. Mitra, A. Gregson, W. Hatfield, and R. Weller, Inorg. Chem. 22, 1729 (1983).

${ }^{7}$ M. Evangelisti, J. Bartolomé, L. J. de Jongh, and G. Filoti, Phys. Rev. B 66, 144410 (2002).

${ }^{8}$ B. Honigmann, H. U. Lenne, and R. Schroedel, Z. Kristallogr. 122, 185 (1965).

${ }^{9}$ C. Ercolani, C. Neri, and P. Porta, Inorg. Chim. Acta 1, 415 (1967).

${ }^{10}$ R. P. Linstead and J. M. Robertson, J. Chem. Soc. 1936, 1736 (1936).

${ }^{11}$ C. G. Barraclough, R. L. Martin, S. Mitra, and R. C. Sherwood, J. Chem. Phys. 53, 1643 (1970).

${ }^{12}$ L. Klemm and W. Klemm, J. Prakt. Chem. 143, 82 (1935).

${ }^{13}$ A. B. P. Lever, J. Chem. Soc. 1965, 1821 (1965).

${ }^{14}$ B. W. Dale, R. J. Williams, C. E. Johnson, and T. L. Thorp, J. Chem. Phys. 49, 3441 (1968).

${ }^{15}$ I. Deszi, A. Balazs, B. Molnar, V. D. Gorobchenko, and L. I. Kukoshevich, J. Inorg. Nucl. Chem. 31, 1661 (1969).

${ }^{16}$ B. W. Dale, Mol. Phys. 28, 503 (1974).

${ }^{17}$ A. Labarta, E. Molins, X. Viñas, J. Tejada, A. Caubet, and S. Alvarez, J. Chem. Phys. 80, 444 (1984).

${ }^{18}$ T. H. Moss and A. B. Robinson, Inorg. Chem. 7, 1692 (1968).

${ }^{19}$ C. G. Baraclough, R. L. Martin, S. Mitra, and R. C. Sherwood, J. Chem. Phys. 53, 1643 (1970).

${ }^{20}$ J. F. Kirner, W. Dow, and W. R. Sheidt, Inorg. Chem. 15, 1685 (1976).

${ }^{21}$ P. Coppens, L. Li, and N. J. Zhu, J. Am. Chem. Soc. 105, 6173 (1983).

${ }^{22}$ T. S. Srivastava, J. L. Przybylinski, and Amar Nath, Inorg. Chem. 13, 1563 (1974).

${ }^{23}$ N. A. Bell, J. S. Brooks, J. K. Robinson, and S. C. Thorpe, J. Chem. Soc., Faraday Trans. 94, 3155 (1998).

${ }^{24}$ D. C. Grenoble and H. G. Drickamer, J. Chem. Phys. 35, 1624 (1971).

${ }^{25}$ J. Silver, P. Luker, A. Houlton, S. Howe, P. Hey, and M. T. Ahmet, J. Mater. Chem. 2(8), 849 (1992).

${ }^{26}$ A. Hudson and H. Whitfield, Inorg. Chem. 6, 1120 (1967).

${ }^{27}$ B. W. Dale, R. J. P. Williams, P. R. Edwards, and C. E. Johnson, J. Chem. Phys. 8, 3445 (1968).
${ }^{28}$ G. T. Yee, B. J. Korte, S. P. Sellers, W. M. Reiff, and C. M. Frommer, Mol. Cryst. Liq. Cryst. Sci. Technol., Sect. A 335, 23 (1999).

${ }^{29}$ W. M. Reiff, C. M. Frommen, G. T. Yee, and S. P. Sellers, Inorg. Chem. 39, 2076 (2000).

${ }^{30}$ M. F. Thomas and C. E. Johnson, in Mössbauer Spectroscopy, edited by D. P. E Dickson and F. J. Berry (Cambridge University Press, Cambridge, England, 1986), p. 148-149.

${ }^{31}$ A. Abragam and B. Bleaney, Electron Paramagnetic Resonance of Transition Ions (Clarendon Press, Oxford, 1970).

${ }^{32}$ D. Sutton, Electronic Spectra of Transition Metal Complexes (McGraw-Hill, London, 1968).

${ }^{33}$ The presence of $s p^{2}$ hybrids is the main distinction of our approach from the original theory of square planar metal complexes elaborated by H. B. Gray and C. J. Ballhausen, J. Am. Chem. Soc. 85, 260 (1963).

${ }^{34}$ W. A. Harrison, Elementary Electronic Structure (World Scientific, Singapore, 1965).

${ }^{35}$ A. E. Clark, B. F. DeSavage, and R. Bozorth, Phys. Rev. 138, A216 (1965).

${ }^{36}$ N. N. Greenwood and T. C. Gibbs, Mössbauer Spectroscopy (Chapman and Hall Ltd., London, 1971).

${ }^{37}$ R. Ingalls, Phys. Rev. 133, A787 (1964).

${ }^{38}$ R. E. Watson and A. J. Freeman, in Hyperfine Interactions, edited by A. J. Freeman (Academic Press, New York, 1967), p. 75.

${ }^{39}$ G. K. Werheim and D. N. E. Buchanan, Phys. Rev. 161, 478 (1967).

${ }^{40}$ H. H. A. Smit, H. J. M de Groot, M. Elmassalami, R. C. Thiel, and L. J. de Jongh, Physica B 154, 237 (1989).

${ }^{41}$ S. P Sellers, B. J. Korte, J. P. Fitzgerald, W. M. Reiff, and G. T. Yee, J. Am. Chem. Soc. 120, 4662 (1998).

${ }^{42}$ G. Filoti, J. Bartolomé, D. P. E. Dickson, C. Rillo, I. Prisecaru, T. Jovmir, V. Kuncser, and C. Turta J. Magn. Magn. Mater. 196197, 561 (1999).

${ }^{43}$ H. Andres, E. L. Bominnar, J. M. Smith, N. A. Eckert, P. L. Holland, and E. Münck, J. Am. Chem. Soc. 124, 3012 (2002).

${ }^{44}$ Q. A. Pankhurst, G. R. Thompson, V. K. Sankaranarayanan, and D. P. E. Dickson, J. Magn. Magn. Mater. 155, 104 (1996).

${ }^{45}$ Q. A. Pankhurst, J. Phys.: Condens. Matter 3, 1323 (1991).

${ }^{46}$ Q. A. Pankhurst and R. J. Pollard, J. Phys.: Condens. Matter 2, 1323 (1990).

${ }^{47}$ E. Van San, E. De Grave, R. E. Vandenberghe, J. Magn. Magn. Mater. 269, 54 (2004).

${ }^{48}$ D. P. E. Dickson, Hyperfine Interact. 113, 15 (1998).

${ }^{49}$ Ö. F. Bakkaloğlu, Q. A. Pankhurst, and M. F. Thomas, J. Phys.: Condens. Matter 5, 3265 (1993).

${ }^{50}$ C. J. Serna, F. Bodker, S. Morup, M. P. Morales, F. Sandiumenge, and S. Veintemillas-Verdaguer, Solid State Commun. 18, 437 (2001).

${ }^{51}$ R. J. Pollard, Q. A. Pankhurst, and P. Zientek, J. Magn. Magn. Mater. 104-107, 1557 (1992). 
${ }^{52}$ H. Miyoshi, J. Phys. Soc. Jpn. 37, 50 (1974).

${ }^{53}$ L. K. Leung, B. J. Evans, and A. H. Morrish, Phys. Rev. B 8, 29 (1973).

${ }^{54}$ F. Harbus and H. E. Stanley, Phys. Rev. B 7, 365 (1973).

${ }^{55}$ In Ref. 7 the susceptibility data was also fitted to the Ising $S$ $=1 / 2$ linear chain model. Unluckily, the fit parameters given there $J / k_{\mathrm{B}}=32.2 \mathrm{~K}, g_{\|}=8.6$, and $g_{\perp}=0.2$ were wrong because of a transcription error in the final manuscript.

${ }^{56}$ M. Elmassalami and L. J. de Jongh, Physica B 154, 254 (1989).

${ }^{57}$ H. J. M. de Groot, L. J. de Jongh, R. C. Thiel, and J. Reedijk, Phys. Rev. B 30, 4041 (1984).

${ }^{58}$ J. L. Dormann, D. Fiorani, and E. Tronc, Adv. Chem. Phys. 98, 283 (1997). 